\title{
Reduction of $\mathrm{Cp}_{2} \mathrm{ZrCl}_{2}$ with mischmetall : A new method for generating an efficient " $\mathrm{Cp}_{2} \mathrm{Zr}$ " equivalent.
}

\author{
Clément Denhez $^{[\mathrm{a}]}$, Sédami Médégan ${ }^{[\mathrm{b}]}$, Florence Hélion ${ }^{[\mathrm{b}]}$, \\ Jean-Louis Namy $^{[b] *}$, Jean-Luc Vasse ${ }^{[a]}$ and Jan Szymoniak ${ }^{[a] *}$
}

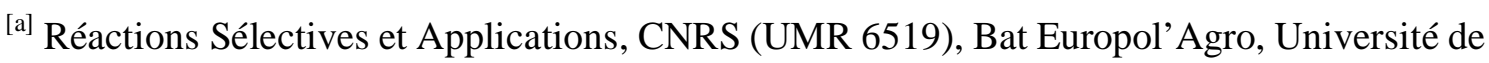
Reims, 51687 Reims Cedex 2, France. Fax: +33-326-913-166

[b] Laboratoire de Catalyse Moléculaire, CNRS (UMR 8182), ICMMO, Bat 420, Université Paris-Sud, 91405, Orsay, France. Fax: +33-169-154-680

Corresponding authors:

jan.szymoniak@univ-reims.fr

jelonamy@icmo.u-psud.fr 


\section{Table of Contents}

Materials and general procedures

Spectroscopic data for new compounds

S4-S9

${ }^{1} \mathrm{H}$ NMR of new compounds

S9-S14 


\section{Materials and general procedures}

All reactions were conducted under an atmosphere of argon using standard Schlenk techniques. Mischmetall ingots purchased from Fluka or Alfa Aesar - Johnson Matthey (minimum rare earth content is $99.0 \%$ ) were powdered with a rasp under Ar. Prior to use, tetrahydrofuran was distilled under argon from sodium benzophenone ketyl, $\mathrm{CH}_{2} \mathrm{Cl}_{2}$ and DMF were distilled under argon from $\mathrm{CaH}_{2} \cdot{ }^{1} \mathrm{H}$ and ${ }^{13} \mathrm{C}$ NMR spectra were recorded in $\mathrm{CDCl}_{3}$ on a Brucker AC-250. Mass spectra were recorded on a Micromass Q-TOF micro MS spectrometer. Infra-Red spectra were recorded on a Nicolet Avatar 320 FT-IR.

\section{Procedure A}

\section{Homocoupling of alkynes}

To a suspension of mischmetall powder $(120 \mathrm{mg})$ in THF $(5 \mathrm{~mL})$ were added the alkyne $(2 \mathrm{mmol})$ and $\mathrm{Cp}_{2} \mathrm{ZrCl}_{2}(1 \mathrm{mmol})$. The resulting mixture was warmed to $50^{\circ} \mathrm{C}$ until a deep red color appears, then the reaction was stirred for 4 hours at r.t. The reaction was quenched by adding an aqueous solution of $\mathrm{HCl}(1 \mathrm{~N}, 2 \mathrm{~mL})$, the aqueous layer was extracted with AcOEt $(2 \times 5 \mathrm{~mL})$, the organic phases were combined, dried over $\mathrm{MgSO}_{4}$, filtered and concentrated under vaccum. The residue was purified by column chromatography on silica gel using a mixture of petroleum ether / AcOEt as eluant.

\section{Procedure $B$}

To a mixture of mischmetall powder $(120 \mathrm{mg}), \mathrm{Cp}_{2} \mathrm{ZrCl}_{2}(1 \mathrm{mmol})$ and the substrate $(1 \mathrm{mmol})$ in THF $(5 \mathrm{~mL})$ were added 2 crystals of $\mathrm{I}_{2}$. The color turned red within $10 \mathrm{~min}$. The reaction mixture was stirred for 4 hours at r.t., then quenched by adding dropwise an aqueous solution of $\mathrm{HCl}(1 \mathrm{~N}, 2 \mathrm{~mL})$. The aqueous layer was extracted with AcOEt $(2 \times 5 \mathrm{~mL})$, the organic phases were combined, dried over $\mathrm{MgSO}_{4}$, filtered and concentrated under vaccum. The residue was purified by column chromatography on silica gel using a mixture of petroleum ether / AcOEt as eluant.

\section{Prodedure C}

\section{Intermolecular coupling of imine and alkyne}

To a suspension of mischmetall powder $(120 \mathrm{mg})$ in THF $(5 \mathrm{~mL})$ were added the imine (1 mmol), $\mathrm{Cp}_{2} \mathrm{ZrCl}_{2}(1 \mathrm{mmol})$, and 2 crystals of $\mathrm{I}_{2}$. The reaction was stirred for 1 hour at r.t. A solution of the alkyne $(1.1 \mathrm{mmol})$ in THF $(2 \mathrm{~mL})$ was then added dropwise and the stirring was continued for 8 additionnal hours. The reaction was quenched by adding dropwise an aqueous solution of $\mathrm{HCl}(1 \mathrm{~N}, 2 \mathrm{~mL})$. The aqueous layer was neutralized with a saturated aqueous solution of $\mathrm{NaHCO}_{3}$, extracted with AcOEt $(2 \times 5 \mathrm{~mL})$. The organic phases were combined, dried over $\mathrm{MgSO}_{4}$, filtered and concentrated under vaccum. The residue was purified by column chromatography on silica gel using a mixture of petroleum ether / AcOEt as eluant.

\section{Intramolecular coupling of imine and alkene and intramolecular coupling of alkyne and alkene}

These reactions were carried out according to procedure $\mathrm{B}$ using the following quantities: mischmetall powder $(120 \mathrm{mg})$, the substrate $(1 \mathrm{mmol})$ and $\mathrm{Cp}_{2} \mathrm{ZrCl}_{2}(1 \mathrm{mmol})$. 
Spectroscopic data for new compounds

(4E,6E)-5,6-dipropyldeca-4,6-diene 1c

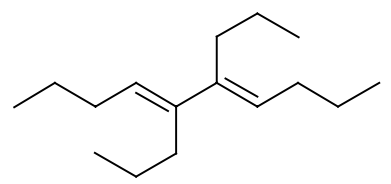

Obtained according to the procedure A. ${ }^{1} \mathrm{H}$ NMR $(200 \mathrm{MHz}) \delta: 0.91(\mathrm{~m}, 12 \mathrm{H}), 1.44(\mathrm{~m}, 8 \mathrm{H})$, $2.09(\mathrm{~m}, 8 \mathrm{H}), 5.35(\mathrm{t}, 2 \mathrm{H}, J=5.3 \mathrm{~Hz}) ;{ }^{13} \mathrm{C} \mathrm{NMR} \delta: 14.0,23.0,31.0,126.4,141.7$; IR $\left(\mathrm{CaF}_{2}\right.$, film), v: 3050, 2958, 2928, 2871, 1729, 1633, 1463, 1376, 1260; MS (IE): m/z : 222 (70\%), $179(95 \%), 95(100 \%)$.

\section{$N$-allyl- $N$-propargyl-4-methoxybenzylamine}

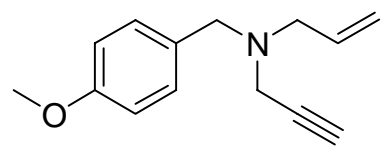

To a mixture of $\mathrm{N}$-allyl- 4-methoxybenzylamine $(1.77 \mathrm{~g}, 10 \mathrm{mmol})$ and potassium carbonate $(2.1 \mathrm{~g}, 15 \mathrm{mmol})$ in DMF $(25 \mathrm{~mL})$ was added dropwise a solution of propargyl bromide in $\operatorname{DMF}(1.3 \mathrm{~g}, 11 \mathrm{mmol})$ at $0^{\circ} \mathrm{C}$. The reaction mixture was stirred for 4 hours at r.t.. Water $(15$ $\mathrm{mL})$ was added and the aqueous layer was extracted with ether $(3 \times 15 \mathrm{~mL})$. The organic layers were combined, washed with water $(2 \times 10 \mathrm{~mL})$ dried over $\mathrm{MgSO}_{4}$, filtered and concentrated under vaccum. The residue was purified by column chromatography on silica gel using a $90: 10$ mixture of petroleum ether : AcOEt as eluant to give the title compound as a yellow oil (79\%). ${ }^{1} \mathrm{H}$ NMR $\delta: 2.23(\mathrm{t}, 1 \mathrm{H}, J=2.3 \mathrm{~Hz}), 3.16(\mathrm{~d}, 2 \mathrm{H}, J=6.6 \mathrm{~Hz}), 3.29(\mathrm{~d}, 2 \mathrm{H}, J$ $=2.3 \mathrm{~Hz}), 3.57(\mathrm{~s}, 2 \mathrm{H}), 3.78(\mathrm{~s}, 3 \mathrm{H}), 5.16(\mathrm{~d}, 1 \mathrm{H}, J=10.2 \mathrm{~Hz}), 5.27(\mathrm{~d}, 1 \mathrm{H}, J=16.7 \mathrm{~Hz}), 5.86$ $(\mathrm{ddt}, 1 \mathrm{H}, J=16.7,10.2,6.5 \mathrm{~Hz}), 6.85(\mathrm{~d}, 2 \mathrm{H}, J=8.7 \mathrm{~Hz}), 7.26(\mathrm{~d}, 2 \mathrm{H}, J=8.7 \mathrm{~Hz}) ;{ }^{13} \mathrm{C}$ NMR $\delta$ : 40.9, 55.1, 56.4, 56.5, 73.1, 78.5, 113.6, 117.9, 130.2, 130.4, 135.5, 158.7 ; IR (film) v: 3295, 2834, 1612, 1512; HRMS-ESI : $m / z$ [M+H] ${ }^{+}$calcd for $\mathrm{C}_{14} \mathrm{H}_{18} \mathrm{NO}: 216.1388$, found: 216.1392 .

\section{$N$-(4-methoxybenzyl)- $N$-allyl-3-trimethylsilylprop-2-ynamine}

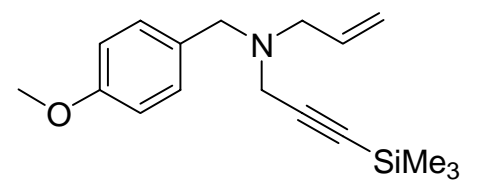

To a solution of $N$-allyl- $N$-propargyl-4-methoxybenzylamine $(2.15 \mathrm{~g}, 10 \mathrm{mmol})$ in THF (25 $\mathrm{mL})$, was added dropwise a solution of butyllithiun $(2.5 \mathrm{M}$, in hexanes, $4 \mathrm{~mL})$ at $-78^{\circ} \mathrm{C}$, and the reaction was stirred at this temperature for $15 \mathrm{~min}$. and warmed to $0^{\circ} \mathrm{C}$. chlorotrimethysilane $(1.4 \mathrm{~mL}, 11 \mathrm{mmol})$ was added dropwise, and the reaction was stirred for 3 hours at r.t. The reaction was quenched with a saturated aqueous solution of $\mathrm{NaHCO}_{3}$, the 
aqueous layer was extracted with AcOEt $(2 \times 15 \mathrm{~mL})$. The organic phases were combined, dried over $\mathrm{MgSO}_{4}$, filtered and concentrated under vaccum to give the title compound as a red oil $(92 \%) .{ }^{1} \mathrm{H}$ NMR $\delta: 0.14(\mathrm{~s}, 9 \mathrm{H}), 2.94(\mathrm{dd}, 2 \mathrm{H}, J=6.5,1.1 \mathrm{~Hz}), 3.07(\mathrm{~s}, 2 \mathrm{H}), 3.35(\mathrm{~s}, 2 \mathrm{H})$, $3.59(\mathrm{~s}, 3 \mathrm{H}), 4.95(\mathrm{dm}, 1 \mathrm{H}, J=10.1 \mathrm{~Hz}), 5.05(\mathrm{dm}, 1 \mathrm{H}, J=16.6 \mathrm{~Hz}), 5.66(\mathrm{tdd}, 1 \mathrm{H}, J=16.6$, $10.1,6.5 \mathrm{~Hz}), 6.64(\mathrm{~d}, 2 \mathrm{H}, J=8.7 \mathrm{~Hz}), 7.05(\mathrm{~d}, 2 \mathrm{H}, J=8.7 \mathrm{~Hz}) ;{ }^{13} \mathrm{C}$ NMR $\delta: 0.12,42.1,55.2$, 56.5, 90.1, 100.9, 113.6, 117.9, 130.3, 130.4, 135.5, 158.6; IR (film) v: 2957, 2833, 1612, 1513; HRMS-ESI : $m / z[\mathrm{M}+\mathrm{H}]^{+}$calcd for $\mathrm{C}_{17} \mathrm{H}_{26} \mathrm{NOSi}$ : 288.1784 , found: 288.1777 .

\section{1-(4-methoxybenzyl)-3-methyl-4-methylenepyrrolidine 4a}

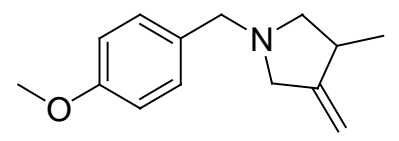

Obtained according to the procedure B. ${ }^{1} \mathrm{H}$ NMR $\delta: 1.10(\mathrm{~d}, 3 \mathrm{H}, J=6.8 \mathrm{~Hz}), 2.05(\mathrm{t}, 1 \mathrm{H}, J=$ $8.6 \mathrm{~Hz}), 2.71(\mathrm{dd}, 1 \mathrm{H}, J=13.0,6.5 \mathrm{~Hz}), 2.95-3.05(\mathrm{~m}, 2 \mathrm{H}), 3.40(\mathrm{~m}, 1 \mathrm{H}), 3.51(\mathrm{~d}, 1 \mathrm{H}, J=$ $11.9 \mathrm{~Hz}), 3.58(\mathrm{~d}, 1 \mathrm{H}, J=11.9 \mathrm{~Hz}), 3.80(\mathrm{~s}, 3 \mathrm{H}), 4.81(\mathrm{q}, J=2.2 \mathrm{~Hz}, 1 \mathrm{H}), 4.87(\mathrm{q}, J=2.2 \mathrm{~Hz}$, $1 \mathrm{H}), 6.85(\mathrm{~d}, 2 \mathrm{H}, J=8.5 \mathrm{~Hz}), 7.25(\mathrm{~d}, 2 \mathrm{H}, J=8.5 \mathrm{~Hz}) ;{ }^{13} \mathrm{C} \mathrm{NMR} \delta: 17.6,37.4,55.2,59.3$, 59.9, 62.1, 103.8, 113.5, 113.6, 129.9, 130.0, 153.9; IR (film) v: 2931, 2835, 1684, 1611; HRMS-ESI : $m / z$ [M+H] $]^{+}$calcd for $\mathrm{C}_{14} \mathrm{H}_{20} \mathrm{NO}: 218.1545$, found: 218.1543 .

\section{(Z)-1-(4-methoxybenzyl)-3-methyl-4-[(trimethylsilyl)methylene]pyrrolidine 4b}

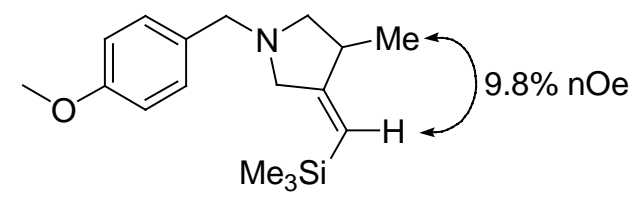

Obtained according to the procedure B. ${ }^{1} \mathrm{H}$ NMR $\delta: 0.05(\mathrm{~s}, 9 \mathrm{H}), 1.05(\mathrm{~d}, 3 \mathrm{H}, J=6.8 \mathrm{~Hz})$, $1.94(\mathrm{t}, 1 \mathrm{H}, J=8.7 \mathrm{~Hz}), 2.64(\mathrm{~m}, 1 \mathrm{H}), 2.90-3.01(\mathrm{~m}, 2 \mathrm{H}), 3.49-3.56(\mathrm{~m}, 2 \mathrm{H}), 3.59(\mathrm{~d}, 1 \mathrm{H}, J=$ $12.8 \mathrm{~Hz}), 3.74(\mathrm{~s}, 3 \mathrm{H}), 5.22(\mathrm{~m}, 1 \mathrm{H}) 6.85(\mathrm{~d}, 2 \mathrm{H}, J=8.6 \mathrm{~Hz}), 7.24(\mathrm{~d}, 2 \mathrm{H}, J=8.6 \mathrm{~Hz}) ;{ }^{13} \mathrm{C}$ NMR $\delta$ : $-0.4,17.3,40.2,55.2,59.3,60.1,61.1,113.5,116.6,129.9,130.9,158.6,162.7$; IR (film) v: 2954, 2777, 1634, 1513; HRMS-ESI: $m / z[\mathrm{M}+\mathrm{H}]^{+}$calcd for $\mathrm{C}_{17} \mathrm{H}_{28} \mathrm{NOSi}$ : 290.1940, found: 290.1938.

\section{$N$-[2-(allyloxy)benzylidene]aniline ${ }^{1}$}<smiles>C=CCOc1ccccc1/C=N\c1ccccc1</smiles>

${ }^{1} \mathrm{H}$ NMR $\delta: 4.62(\mathrm{~d}, 2 \mathrm{H}, J=5.1 \mathrm{~Hz}), 5.30(\mathrm{ddd}, 1 \mathrm{H}, J=10.4,2.6,1.5 \mathrm{~Hz}), 5.42(\mathrm{ddd}, 1 \mathrm{H}, J=$ $17.3,2.6,1.5 \mathrm{~Hz}), 6.07(\mathrm{tdd}, 1 \mathrm{H}, J=17.3,10.4,5.1 \mathrm{~Hz}), 6.94(\mathrm{~d}, 1 \mathrm{H}, J=8.3 \mathrm{~Hz}), 7.05(\mathrm{t}, 1 \mathrm{H}$, $J=7.8 \mathrm{~Hz}), 7.21(\mathrm{~m}, 3 \mathrm{H}), 7.39(\mathrm{~m}, 3 \mathrm{H}), 8.17(\mathrm{~d}, 1 \mathrm{H}, J=7.7 \mathrm{~Hz}), 8.96(\mathrm{~s}, 1 \mathrm{H})$.

\footnotetext{
${ }^{1}$ Black, M.; Cadogan, J. I. G.; Leardini, R.; McNab, H.; McDougald, G.; Nanni, D.; Reed, D.; Zompatori, A. J. Chem. Soc., Perkin Trans. 1 1998, 11, 1825-1832.
} 


\section{3-Methyl-4-phenylaminochroman 5a}<smiles>CC1COc2ccccc2C1Nc1ccccc1</smiles>

Obtained according to the procedure $\mathrm{B}$ and isolated as a $1: 1$ mixture of diastereomers. ${ }^{1} \mathrm{H}$ NMR $\delta: 1.02\left(\mathrm{~d}, 3 \mathrm{H}^{\beta}, J=7.0 \mathrm{~Hz}\right), 1.09\left(\mathrm{~d}, 3 \mathrm{H}^{\alpha}, J=7.1 \mathrm{~Hz}\right), 2.22\left(\mathrm{ddd}, 1 \mathrm{H}^{\beta}, J=11.3,4.3,2.6\right.$ $\mathrm{Hz}$ ), 2.42 (ddd, $\left.1 \mathrm{H}^{\alpha}, J=7.0,4.8,2.8 \mathrm{~Hz}\right), 3.73\left(\mathrm{br} \mathrm{d}, 1 \mathrm{H}^{\beta}, J=8.5 \mathrm{~Hz}\right), 3.94-4.06\left(\mathrm{~m}, 1 \mathrm{H}^{\alpha}\right.$, $\left.1 \mathrm{H}^{\beta}\right), 4.14-4.27\left(\mathrm{~m}, 2 \mathrm{H}^{\alpha}, 1 \mathrm{H}^{\beta}\right), 4.77\left(\mathrm{dd}, 1 \mathrm{H}^{\beta}, J=8.5,4.8 \mathrm{~Hz}\right), 6.63-7.20(\mathrm{~m}, 9 \mathrm{H}) ;{ }^{13} \mathrm{C} \mathrm{NMR}$ $\delta$ : $11.4,14.3,30.5,31.5,51.2,53.3,67.6,68.9,112.4,113.1,116.5,116.6,117.3,117.5$, 120.6, 120.7, 122.2, 123.5, 128.7, 128.9, 129.3, 129.4, 130.6, 146.7, 147.6, 153.9, 154.274; IR (film) v: 3395, 2964, 2873, 1733; HRMS-ESI: $m / z$, $[\mathrm{M}+\mathrm{H}]^{+}$calcd for $\mathrm{C}_{16} \mathrm{H}_{18} \mathrm{NO}: 240.1388$; found: 240.1383 .

\section{2-(but-3-enoxy)benzaldehyde ${ }^{2}$}<smiles>C=CCCOc1ccccc1C=O</smiles>

To a suspension of salicylaldehyde $(1.22 \mathrm{~g}, 10 \mathrm{mmol})$, potassium carbonate $(2 \mathrm{~g}, 15 \mathrm{mmol})$ in dry acetone was added 4-Bromobut-1-ene (1.5 g, $11 \mathrm{mmol})$. This suspension was refluxed for 6 hours, cooled to r.t., filtered, and concentrated under vaccum. The residue was purified by column chromatography on silica gel using a $90: 5$ mixture of petroleum ether : AcOEt as eluant to give the title compound as a colourless oil $(62 \%)$. ${ }^{1} \mathrm{H}$ NMR $\delta: 2.61(\mathrm{q}, 2 \mathrm{H}, J=6.5$ $\mathrm{Hz}), 4.14(\mathrm{t}, 2 \mathrm{H}, J=6.5 \mathrm{~Hz}), 5.14(\mathrm{dm}, 1 \mathrm{H}, J=10.0 \mathrm{~Hz}), 5.20(\mathrm{dm}, 1 \mathrm{H}, J=18.2 \mathrm{~Hz}), 5.92$ $(\mathrm{ddd}, 1 \mathrm{H}, J=17.3,10.0,6.5 \mathrm{~Hz}), 6.98(\mathrm{~d}, 1 \mathrm{H}, J=8.2 \mathrm{~Hz}), 7.04(\mathrm{t}, 1 \mathrm{H}, J=7.5 \mathrm{~Hz}), 7.54(\mathrm{td}$, $1 \mathrm{H}, J=7.5,1.8 \mathrm{~Hz}), 7.83(\mathrm{dd}, 1 \mathrm{H}, J=7.7,1.8 \mathrm{~Hz}), 10.51(\mathrm{~s}, 1 \mathrm{H}) ;{ }^{13} \mathrm{C}$ NMR $\delta: 33.5,67.6$, 112.5, 117.5, 120.6, 124.9, 128.2, 133.96, 135.9, 161.2, 189.8; IR (film) v:3078, 2861, 1688, 1599; HRMS-ESI: $m / z$ [M+Na] ${ }^{+}$calcd for $\mathrm{C}_{11} \mathrm{H}_{12} \mathrm{O}_{2} \mathrm{Na}$ : 199.0735 ; found: 199.0741 .

\section{$N$-[2-(but-3-enyloxy)benzylidene]aniline}<smiles>C=CCCOc1ccccc1/C=N\c1ccccc1</smiles>

A solution of 2-(but-3-enyloxy)benzaldehyde $(1.76 \mathrm{~g}, 10 \mathrm{mmol})$ and aniline $(0.93 \mathrm{~g}, 10$ $\mathrm{mmol})$ in $\mathrm{CH}_{2} \mathrm{Cl}_{2}(20 \mathrm{~mL})$ was refluxed for 2 hours in the presence of $4 \AA$ molecular sieve (3 g). The solid residue was filtered off and the filtrate was concentrated under vaccum to give

\footnotetext{
${ }^{2}$ Garanti, L.; Sala, A.; Zecchi, G. J. Org. Chem. 1975, 40, 2403-2406.
} 
the title compound as a pale green oil (79\%). ${ }^{1} \mathrm{H}-\mathrm{NMR} \delta: 2.58$ (qt, $2 \mathrm{H}, J=6.7,1.3 \mathrm{~Hz}$ ), 4.10 $(\mathrm{t}, 2 \mathrm{H}, J=6.7 \mathrm{~Hz}), 5.15(\mathrm{dm}, 2 \mathrm{H}, J=10.2 \mathrm{~Hz}), 5.17(\mathrm{dq}, 2 \mathrm{H}, J=10.2,1.5 \mathrm{~Hz}), 5.90(\mathrm{tdd}, 1 \mathrm{H}$, $J=17.2,10.2,6.7 \mathrm{~Hz}), 6.93(\mathrm{~d}, 1 \mathrm{H}, J=8.2 \mathrm{~Hz}), 7.03(\mathrm{t}, 1 \mathrm{H}, J=7.5 \mathrm{~Hz}), 7.22(\mathrm{~m}, 3 \mathrm{H}), 7.39$ $(\mathrm{m}, 3 \mathrm{H}), 8.15(\mathrm{dd}, 1 \mathrm{H}, J=7.7,1.8 \mathrm{~Hz}), 8.93(\mathrm{~s}, 1 \mathrm{H}) ;{ }^{13} \mathrm{C}$ NMR $\delta: 33.6,67.6,112.1,117.3$, 120.9, 121.0, 124.9, 125.6, 127.5, 129.0, 132.6, 134.2, 152.8, 156.5, 158.8; IR (film) v: 3080, 2925, 1620, 1590, 1250, 760; HRMS-ESI: $\mathrm{m} / z$ [M+H $]^{+}$calcd for $\mathrm{C}_{17} \mathrm{H}_{18} \mathrm{NO}$ : 252.1388; found: 252.1384 .

\section{4-Methyl-5-phenylamino-2,3,4,5-tetrahydrobenzo[b]oxepin $5 b$}<smiles>CC1CCOc2ccccc2C1Nc1ccccc1</smiles>

Obtained according to the procedure B. ${ }^{1} \mathrm{H}$ NMR $\delta: 0.97(\mathrm{~d}, 3 \mathrm{H}, J=7.1 \mathrm{~Hz}), 1.50(\mathrm{dm}, 1 \mathrm{H}, J$ $=15.0 \mathrm{~Hz}), 2.35(\mathrm{~m}, 1 \mathrm{H}), 2.43(\mathrm{dt}, 1 \mathrm{H}, J=15.0,4.2 \mathrm{~Hz}), 3.99(\mathrm{td}, 1 \mathrm{H}, J=12.1,1.9 \mathrm{~Hz}), 4.19$ $(\mathrm{dt}, 1 \mathrm{H}, J=12.1,3.9 \mathrm{~Hz}), 4.41(\mathrm{~d}, 1 \mathrm{H}, J=5.6 \mathrm{~Hz}), 4.51(\mathrm{br} \mathrm{s}, 1 \mathrm{H}), 6.62(\mathrm{dd}, 2 \mathrm{H}, J=8.6,1.0$ $\mathrm{Hz}), 6.65$ (ddd, $1 \mathrm{H}, J=7.1,2.1,1.1 \mathrm{~Hz}), 6.99(\mathrm{~m}, 2 \mathrm{H}), 7.16(\mathrm{~m}, 4 \mathrm{H}) ;{ }^{13} \mathrm{C} \mathrm{NMR} \delta: 17.1,31.6$, $32.4,62.2,68.4,113.5,117.2,121.9,123.9,128.7,129.2,130.1,133.6,147.1,157.9$; IR (film) v: $3421,2963,1582,1459,764,701$; HRMS-ESI: $\mathrm{m} / z[\mathrm{M}+\mathrm{H}]^{+}$calcd for $\mathrm{C}_{17} \mathrm{H}_{20} \mathrm{NO}$ : 254.1545; found: 254.1540 .

\section{(E)-N-(1,3-diphenylallyl)-4-methoxyaniline 6a}<smiles>COc1ccc(NC(/C=C/c2ccccc2)c2ccccc2)cc1</smiles>

Obtained according to the procedure B from $N$-benzylidene-4-methoxybenzenamine $(211 \mathrm{mg}$, $1 \mathrm{mmol})$ and ethynylbenzene $(123 \mu \mathrm{l}, 1.1 \mathrm{mmol}) .{ }^{1} \mathrm{H}$ NMR $\delta: 3.69(\mathrm{~s}, 3 \mathrm{H}), 3.85$ (br s, $\left.1 \mathrm{H}\right)$, $4.99(\mathrm{~d}, 1 \mathrm{H}, J=6.2 \mathrm{~Hz}), 6.37(\mathrm{dd}, 1 \mathrm{H}, J=15.8,6.2 \mathrm{~Hz}), 6.58(\mathrm{~d}, 2 \mathrm{H}, J=8.8 \mathrm{~Hz}), 6.61(\mathrm{~d}, 1 \mathrm{H}$, $J=15.8 \mathrm{~Hz}), 6.73(\mathrm{~d}, 2 \mathrm{H}, J=8.9 \mathrm{~Hz}), 7.28(\mathrm{~m}, 10 \mathrm{H}) ;{ }^{13} \mathrm{C} \mathrm{NMR} \delta: 55.6,61.5,114.7,114.8$, 126.4, 127.1, 127.4, 127.6, 128.5, 130.8, 131.1, 136.6, 141.4, 142.3, 152.2 ; IR (film) v: 3396, 3026, 2831, 1511; HRMS-ESI: $\mathrm{m} / z[\mathrm{M}+\mathrm{H}]^{+}$calcd for $\mathrm{C}_{22} \mathrm{H}_{22} \mathrm{NO}: 316.1701$; found: 316.1693 .

\section{(E)-N-(1-phenyloct-2-enyl)-4-methoxyaniline 6b}<smiles>CCCCC/C=C/C(Nc1ccc(OC)cc1)c1ccccc1</smiles> 
Obtained according to the procedure B from $N$-benzylidene-4-methoxybenzenamine $(211 \mathrm{mg}$, $1 \mathrm{mmol})$ and hept-1-yne $(147 \mu \mathrm{l}, 1.1 \mathrm{mmol}) .{ }^{1} \mathrm{H} \mathrm{NMR} \delta: 0.86(\mathrm{t}, 3 \mathrm{H}, J=6.6 \mathrm{~Hz}), 1.26(\mathrm{~m}$, $6 \mathrm{H}), 2.02(\mathrm{~m}, 2 \mathrm{H}), 3.68(\mathrm{br} \mathrm{s}, 1 \mathrm{H}), 3.70(\mathrm{~s}, 3 \mathrm{H}), 4.79(\mathrm{~d}, 1 \mathrm{H}, J=5.2 \mathrm{~Hz}), 5.60(\mathrm{dd}, 1 \mathrm{H}, J=$ $15.3,6.1 \mathrm{~Hz}), 5.67(\mathrm{dt}, 1 \mathrm{H}, J=15.3,6.2 \mathrm{~Hz}), 6.53(\mathrm{~d}, 2 \mathrm{H}, J=8.9 \mathrm{~Hz}), 6.71(\mathrm{~d}, 2 \mathrm{H}, J=8.9$ $\mathrm{Hz}), 7.29(\mathrm{~m}, 5 \mathrm{H}) ;{ }^{13} \mathrm{C}$ NMR $\delta: 14.0,22.5,28.8,31.5,32.2,55.7,61.3,114.6,114.8,126.9$, 127.0, 128.5, 131.5, 132.7, 141.6, 143.0, 152.0; IR (film) v: 3403, 2929, 2855, 1511; HRMSESI: $m / z[\mathrm{M}+\mathrm{H}]^{+}$calcd for $\mathrm{C}_{21} \mathrm{H}_{28} \mathrm{NO}: 310.2171$; found: 310.2160 . 
${ }^{1} \mathrm{H}$ NMR $250 \mathrm{MHz}$ of new compounds

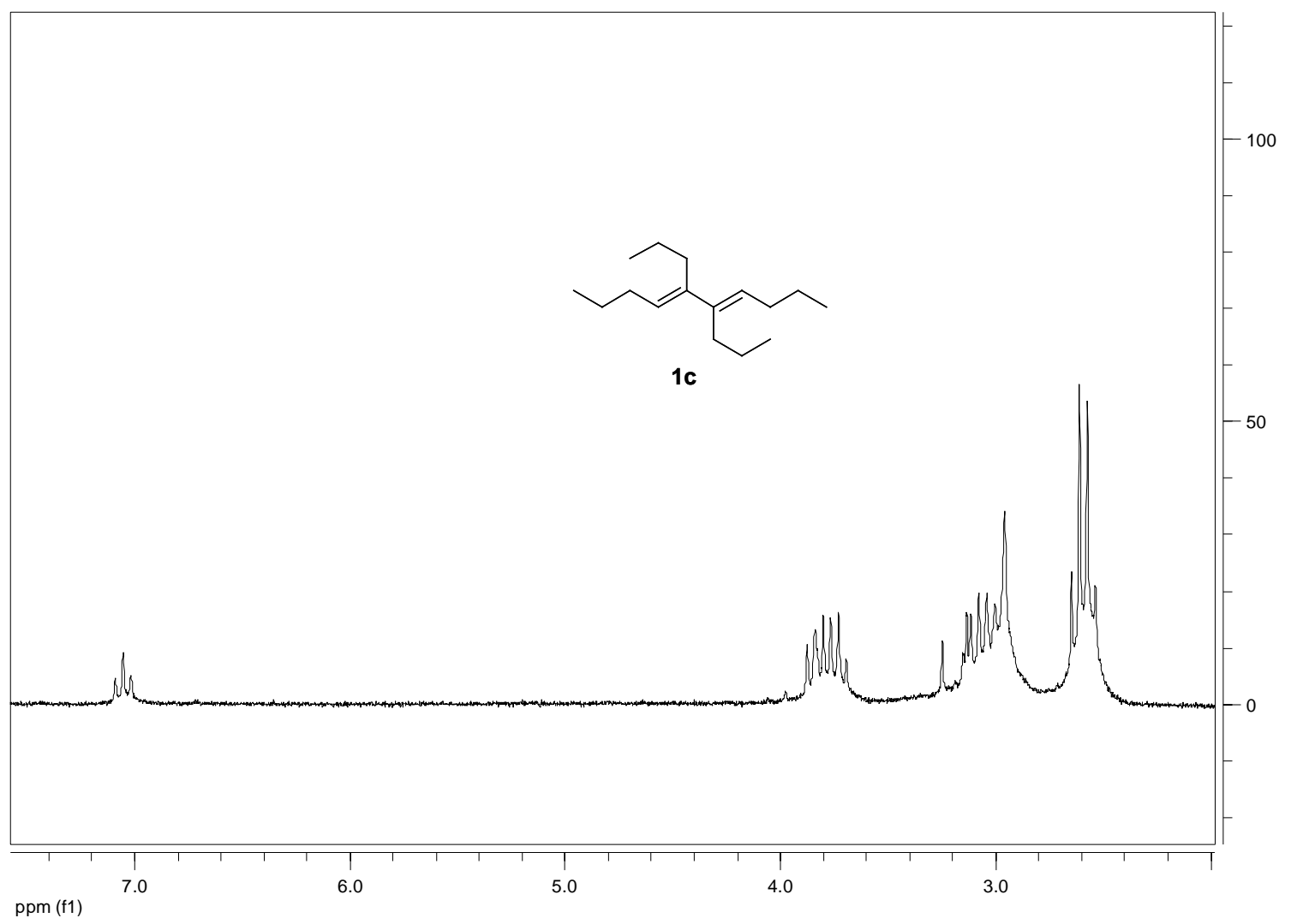



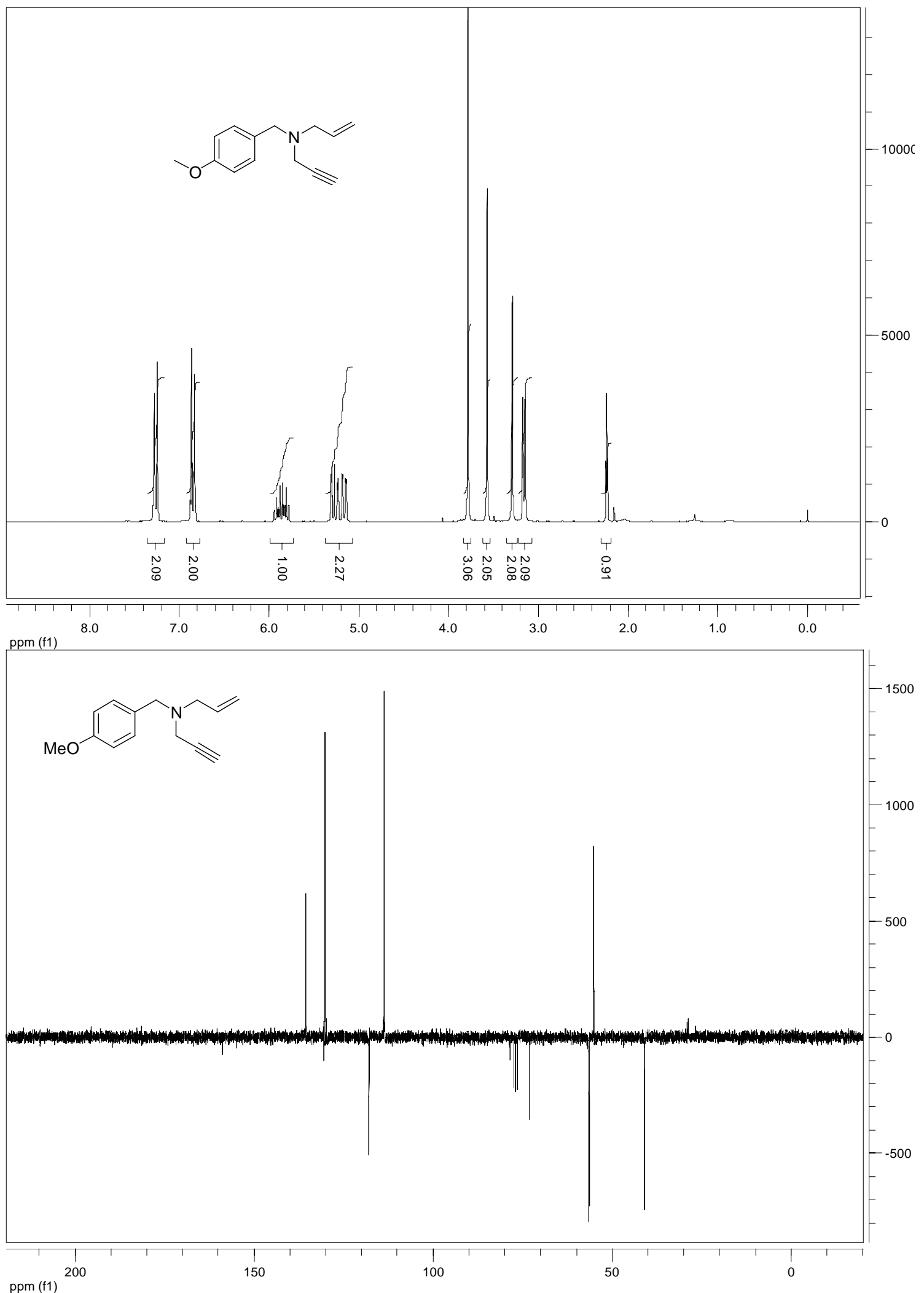

ppm (f1) 

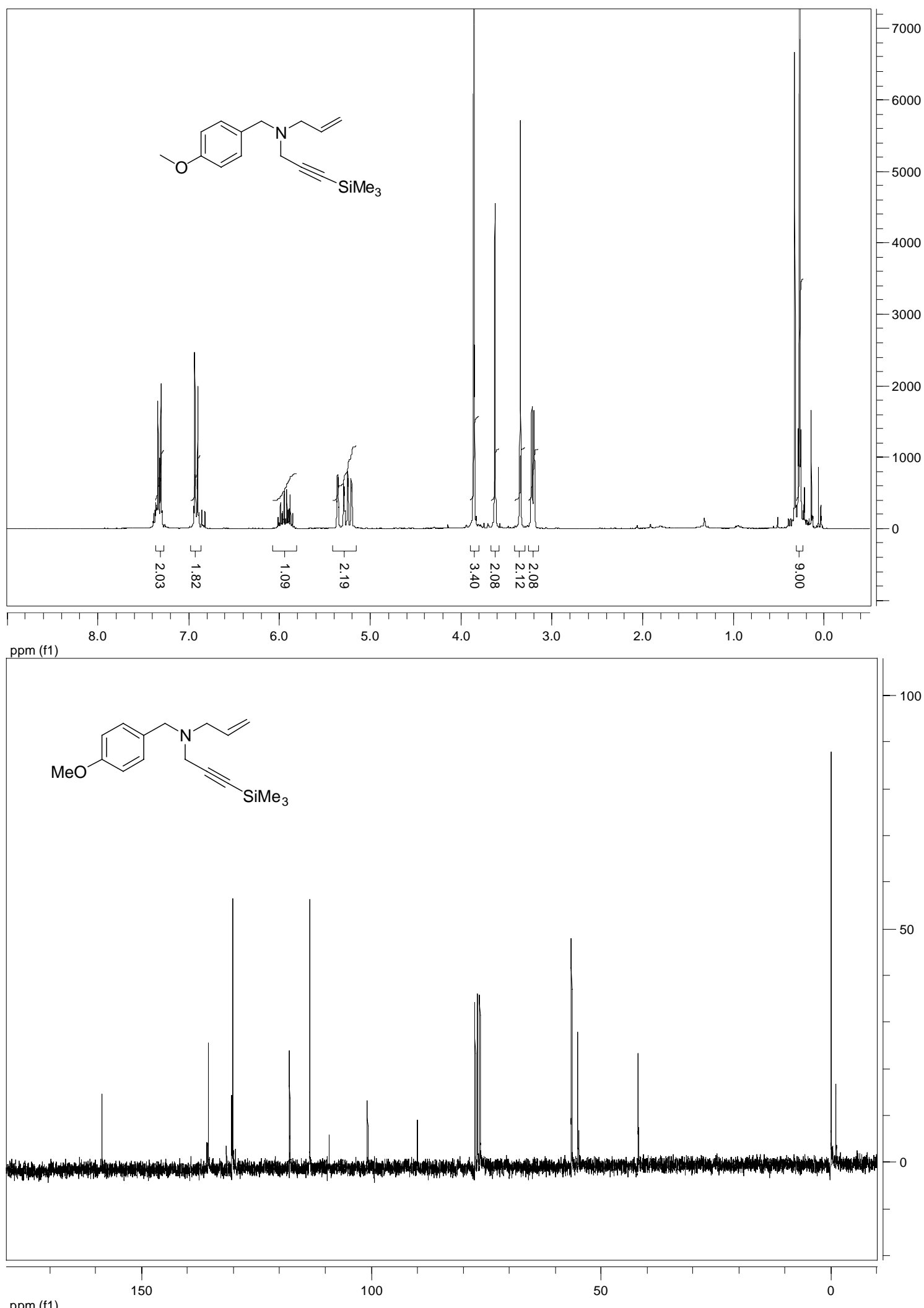



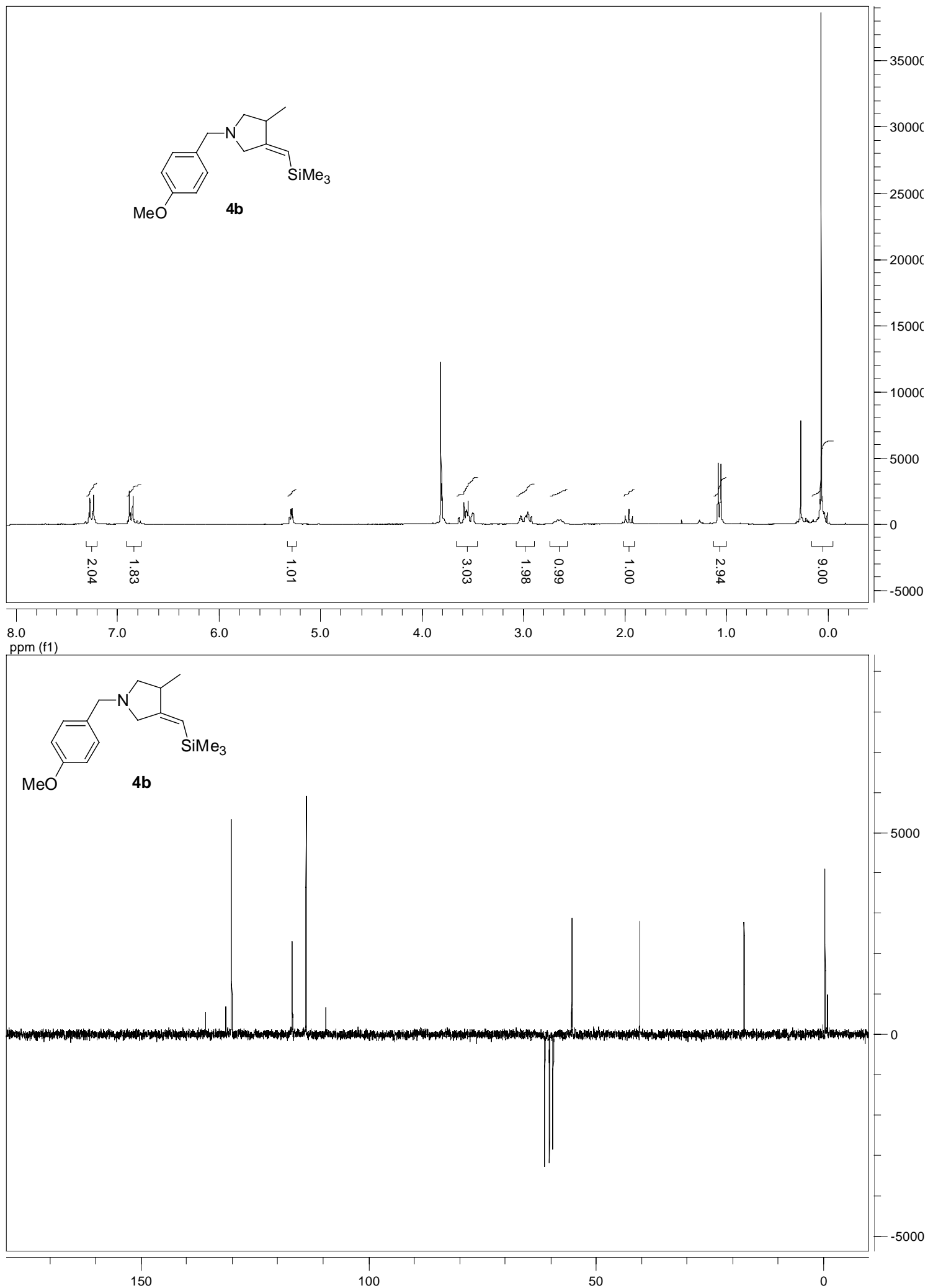

ppm (f1) 

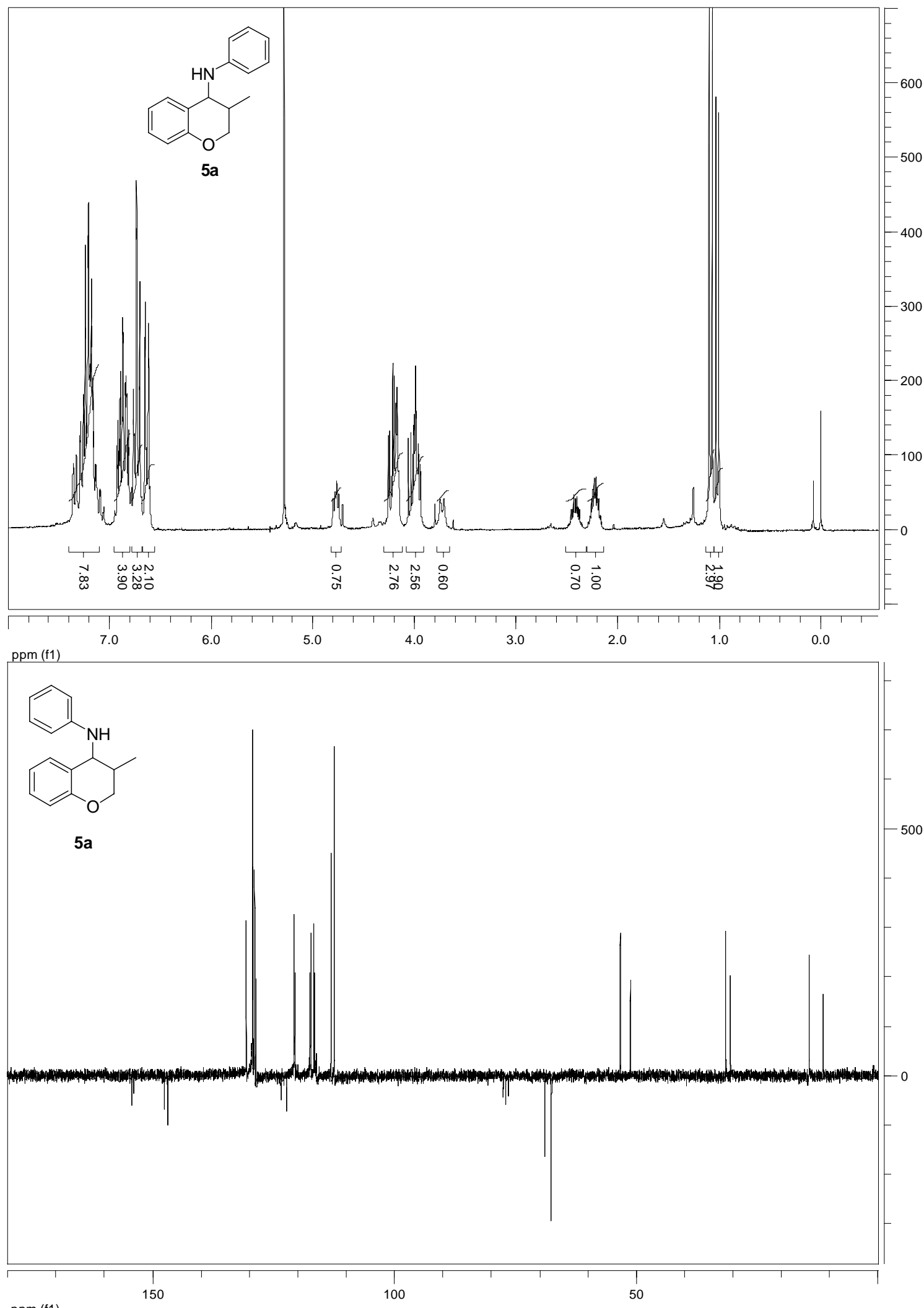

ppm (f1) 

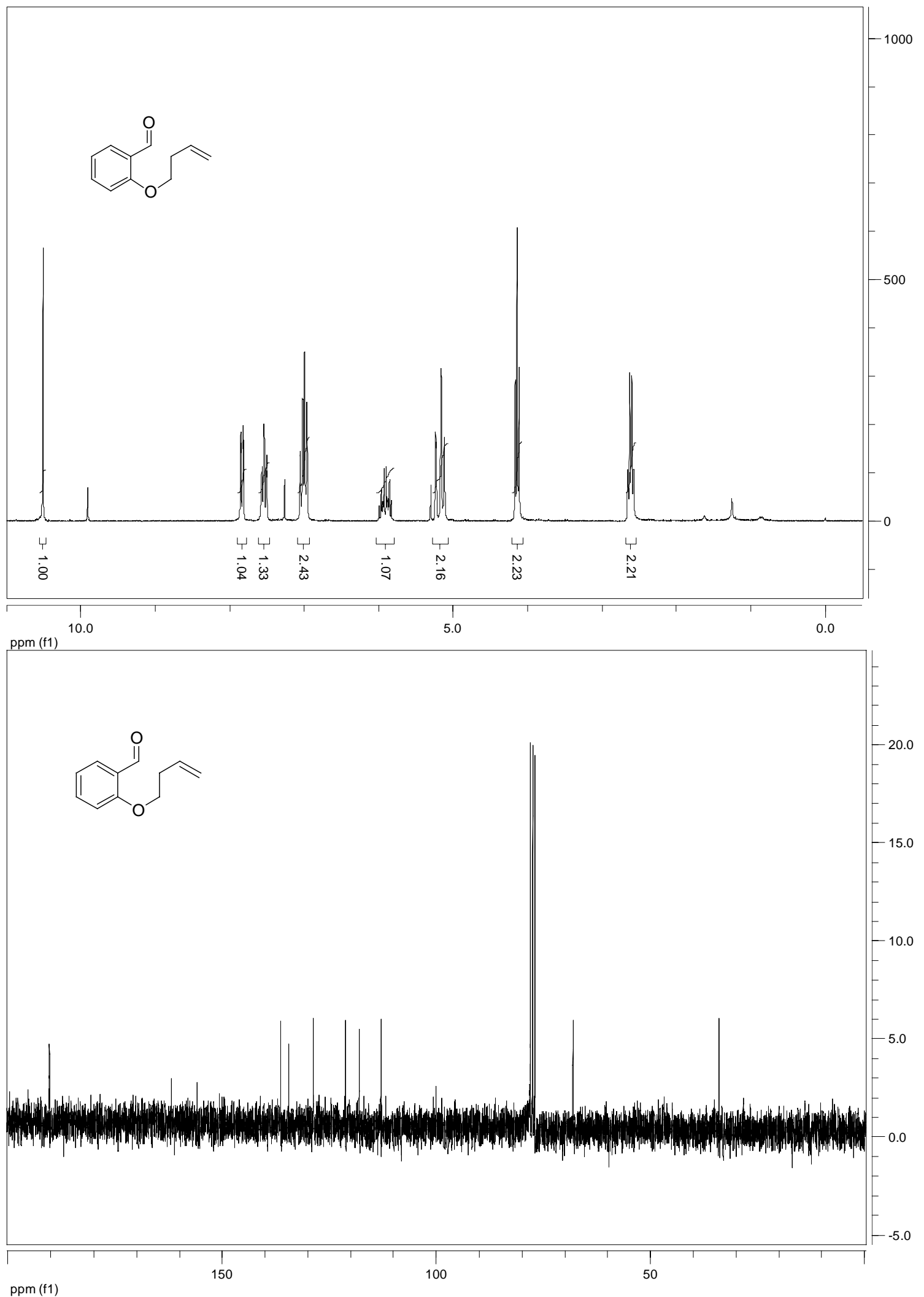


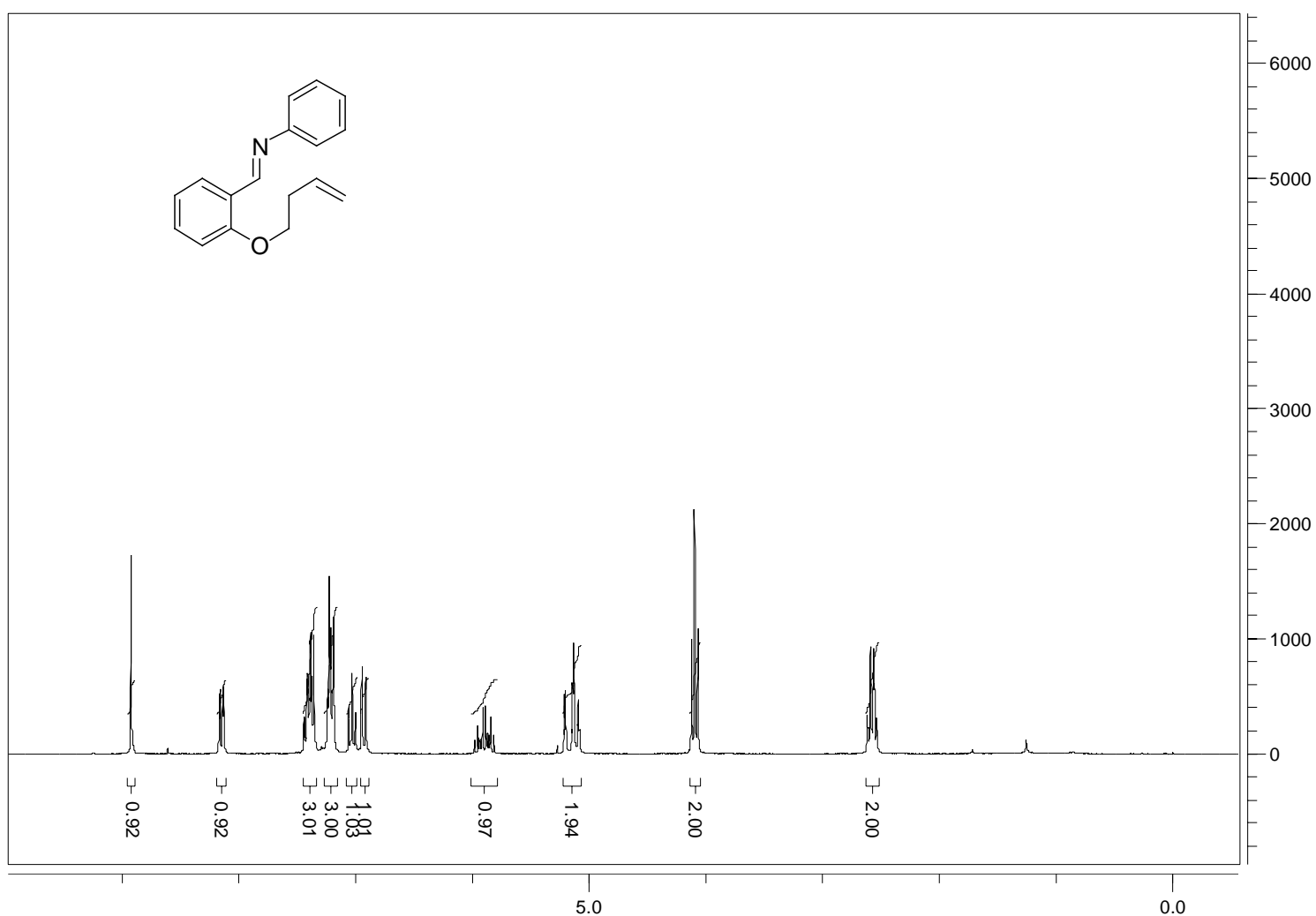

ppm (f1)

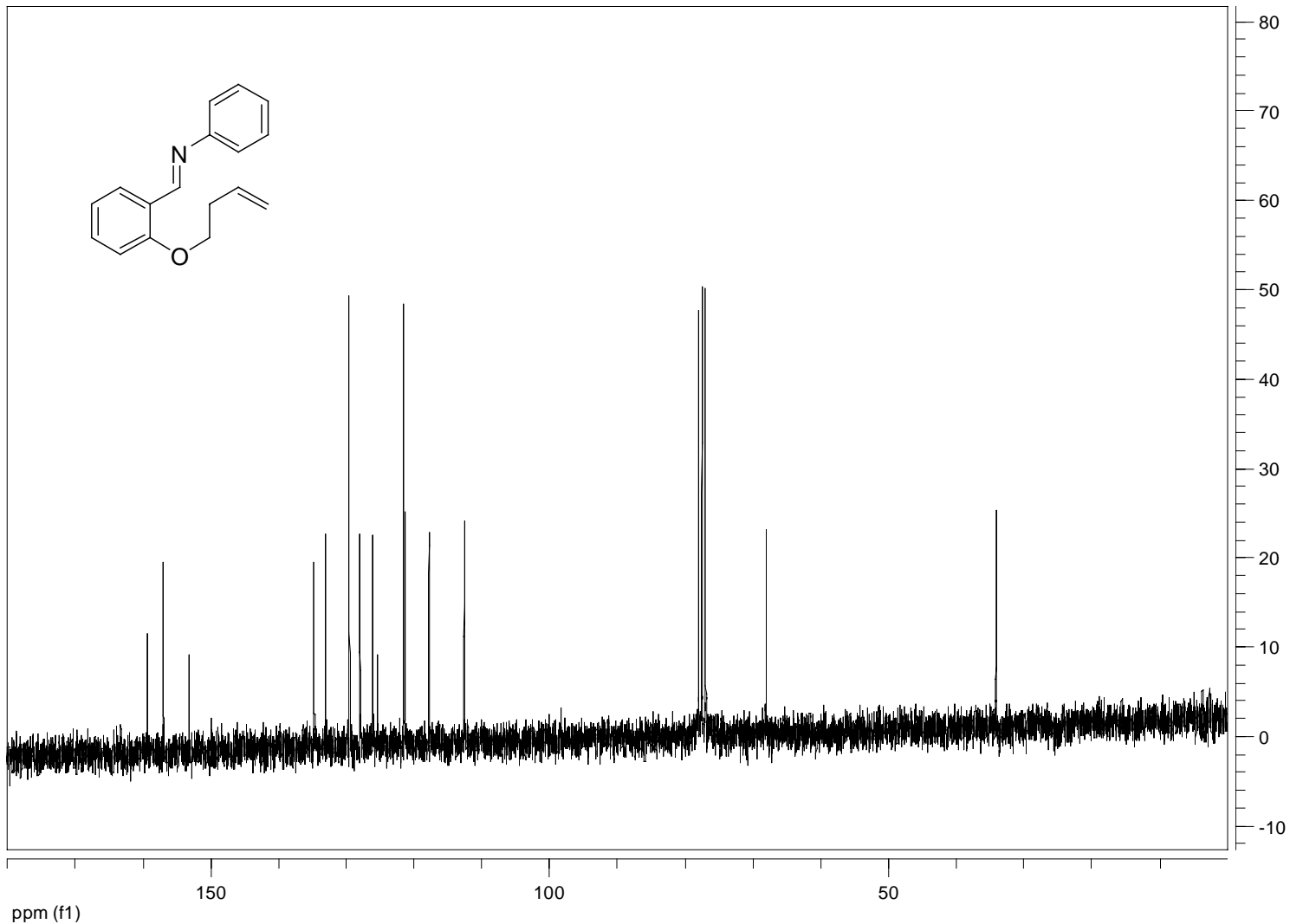



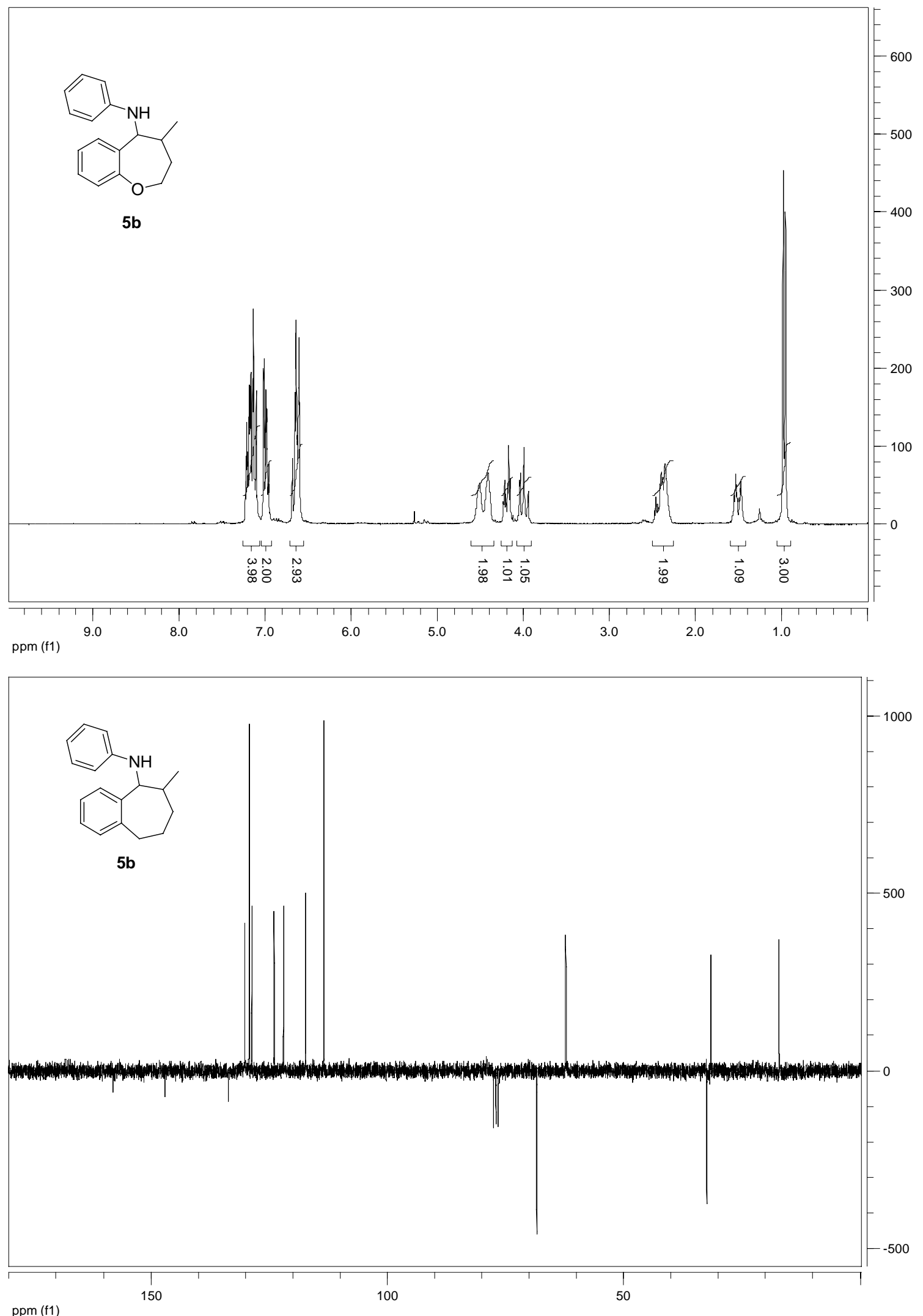


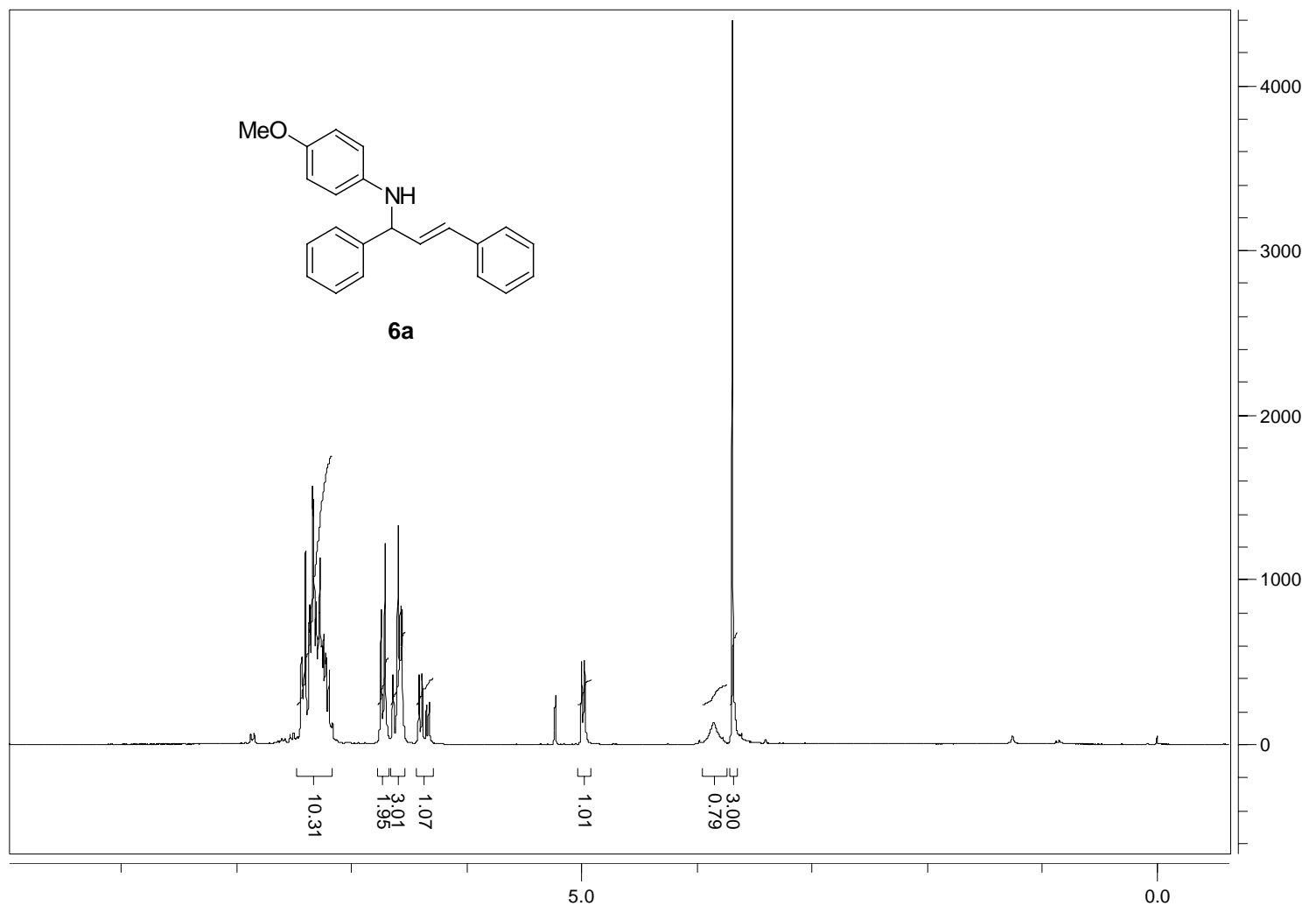

ppm (f1)

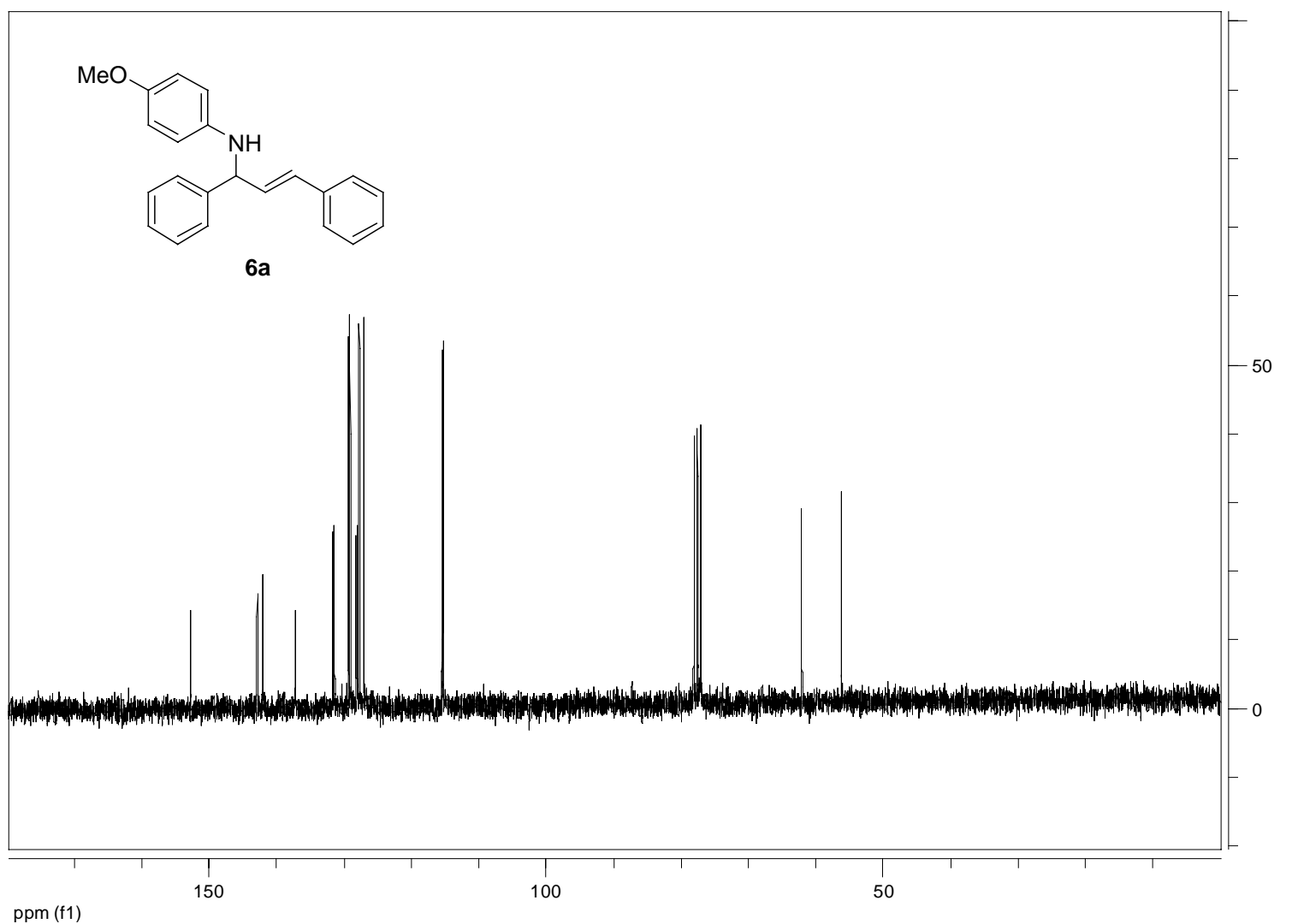

ppm (f1) 


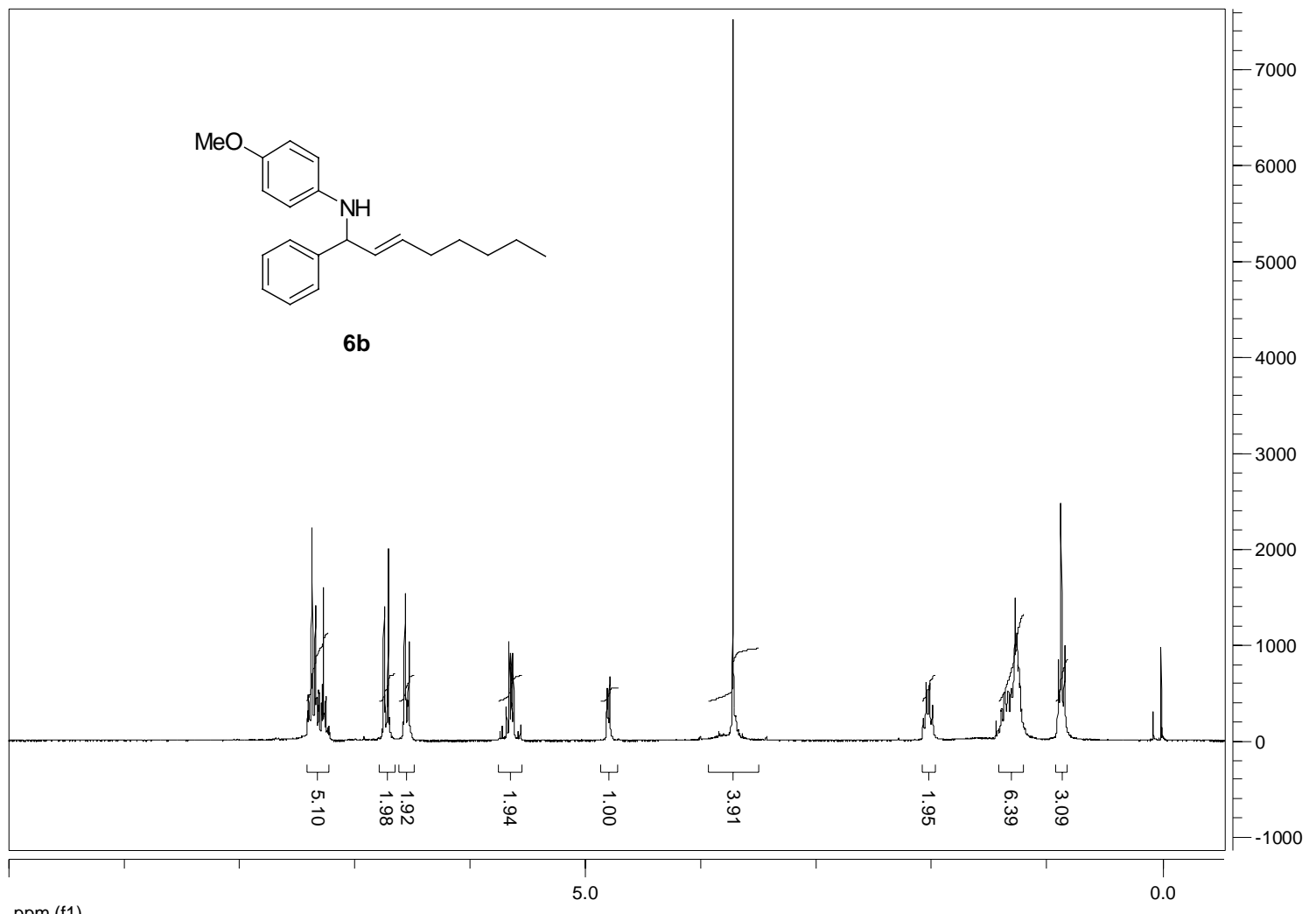

ppm (f1)

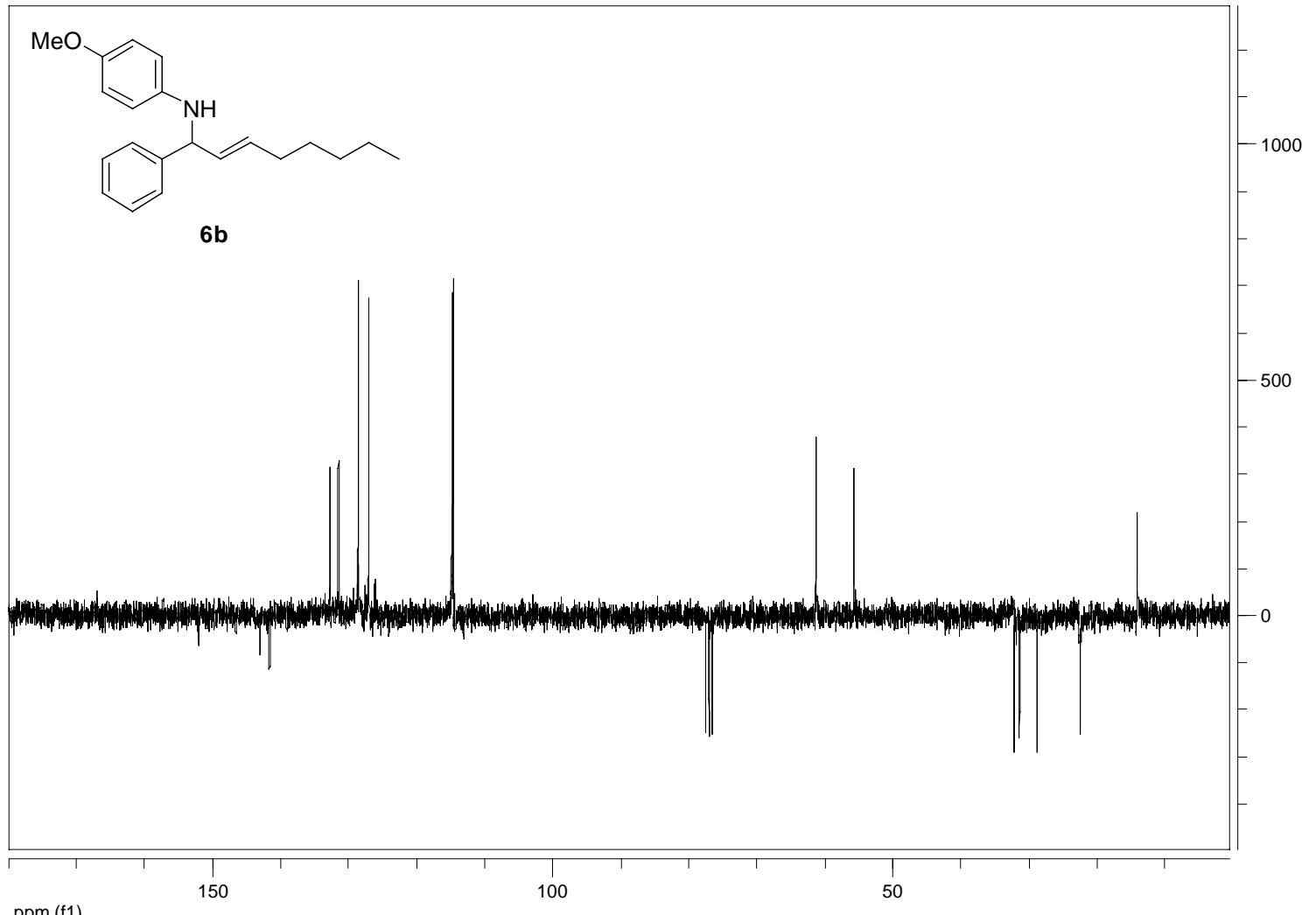

